\title{
The art of building bone: emerging role of chondrocyte-to- osteoblast transdifferentiation in endochondral ossification
}

\author{
Patrick Aghajanian ${ }^{1}$ and Subburaman Mohan ${ }^{1,2,3,4}$
}

\begin{abstract}
There is a worldwide epidemic of skeletal diseases causing not only a public health issue but also accounting for a sizable portion of healthcare expenditures. The vertebrate skeleton is known to be formed by mesenchymal cells condensing into tissue elements (patterning phase) followed by their differentiation into cartilage (chondrocytes) or bone (osteoblasts) cells within the condensations. During the growth and remodeling phase, bone is formed directly via intramembranous ossification or through a cartilage to bone conversion via endochondral ossification routes. The canonical pathway of the endochondral bone formation process involves apoptosis of hypertrophic chondrocytes followed by vascular invasion that brings in osteoclast precursors to remove cartilage and osteoblast precursors to form bone. However, there is now an emerging role for chondrocyte-to-osteoblast transdifferentiation in the endochondral ossification process. Although the concept of "transdifferentiation" per se is not recent, new data using a variety of techniques to follow the fate of chondrocytes in different bones during embryonic and post-natal growth as well as during fracture repair in adults have identified three different models for chondrocyte-to-osteoblast transdifferentiation (direct transdifferentiation, dedifferentiation to redifferentiation, and chondrocyte to osteogenic precursor). This review focuses on the emerging models of chondrocyte-to-osteoblast transdifferentiation and their implications for the treatment of skeletal diseases as well as the possible signaling pathways that contribute to chondrocyte-to-osteoblast transdifferentiation processes.
\end{abstract}

Bone Research (2018)6:19; https://doi.org/10.1038/s41413-018-0021-z

\section{INTRODUCTION}

The process of cell differentiation is a widely studied phenomenon which is the basis for all developmental processes. The basic underlying principle of how cell differentiation proceeds is that with each step of the differentiation pathway, cells become programmed to follow a certain specified lineage progression until they are terminally differentiated with an end point of apoptosis or cell death. ${ }^{1-3}$ Recently, however, many studies have introduced the idea of transdifferentiation, the differentiation of cells (terminally differentiated or not) to a cell type that does not follow the normal, preprogrammed differentiation mechanism. Transdifferentiation refers to a process where one mature cell switches its phenotype and function to that of another mature differentiated cell type. ${ }^{4-8}$ This process occurs via two main mechanisms. The first is via direct transdifferentiation of one tissue type to another without undergoing an intermediate pluripotent state or becoming a progenitor cell, which will be denoted as direct transdifferentiation. The second major method occurs via an intermediate step, often manifested by a dedifferentiation and redifferentiation. This mechanism will be denoted as intermediate transdifferentiation.

Cell transdifferentiation has been described in the literature in multiple tissue types and model organisms, and is therefore neither a species- nor tissue-specific phenomenon. There are now many examples of this phenomenon, but for this review, we will only use a small representative population. In the Drosophila intestine, Takashima et al. have shown that ectodermally derived hindgut cells migrate anteriorly to the midgut to form epithelial cells of the endodermally derived midgut, becoming indistinguishable from the surrounding epithelial cells. ${ }^{9}$ In zebrafish, during normal myocardial regeneration, Zhang et al. observed that a population of atrial cardiomyocytes can migrate to the ventricle and repair it by direct atrial-to-ventricular transdifferentiation and that this mechanism is regulated by notch signaling. ${ }^{10}$ Not surprisingly, tissue regeneration in amphibians and reptiles can also use transdifferentiation mechanisms. Xenopus eye lenses, when removed, can regenerate as the result of transdifferentiation of corneal epithelium to lens cells. ${ }^{11}$ Further studies have shown that upregulation of BMP and WNT ${ }^{12}$ as well as matrix metalloproteases ${ }^{13}$ are required for this direct transdifferentiation.

Furthermore, there are examples of chondrogenic tissues transdifferentiating into cell types of different origins and vice versa. Atherosclerotic lesions in mice have presented what seems to be the transdifferentiation of vascular smooth muscle to chondrogenic tissue. This was confirmed by the reduced expression of a-smooth muscle actin and the increased expression of SOX-9, a marker for immature chondrocytes, in the calcified lesions, ${ }^{14}$ as well as a different study that documented this event to occur via increased expression of tissue non-specific alkaline

\footnotetext{
${ }^{1}$ Musculoskeletal Disease Center, Veterans Affairs Loma Linda Healthcare System, Loma Linda, California, USA; ${ }^{2}$ Department of Medicine, Loma Linda University, Loma Linda,

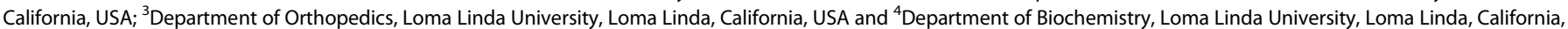
USA

Correspondence: Subburaman Mohan (Subburaman.mohan@va.gov)
}

Received: 28 September 2017 Revised: 26 April 2018 Accepted: 2 May 2018

Published online: 14 June 2018 


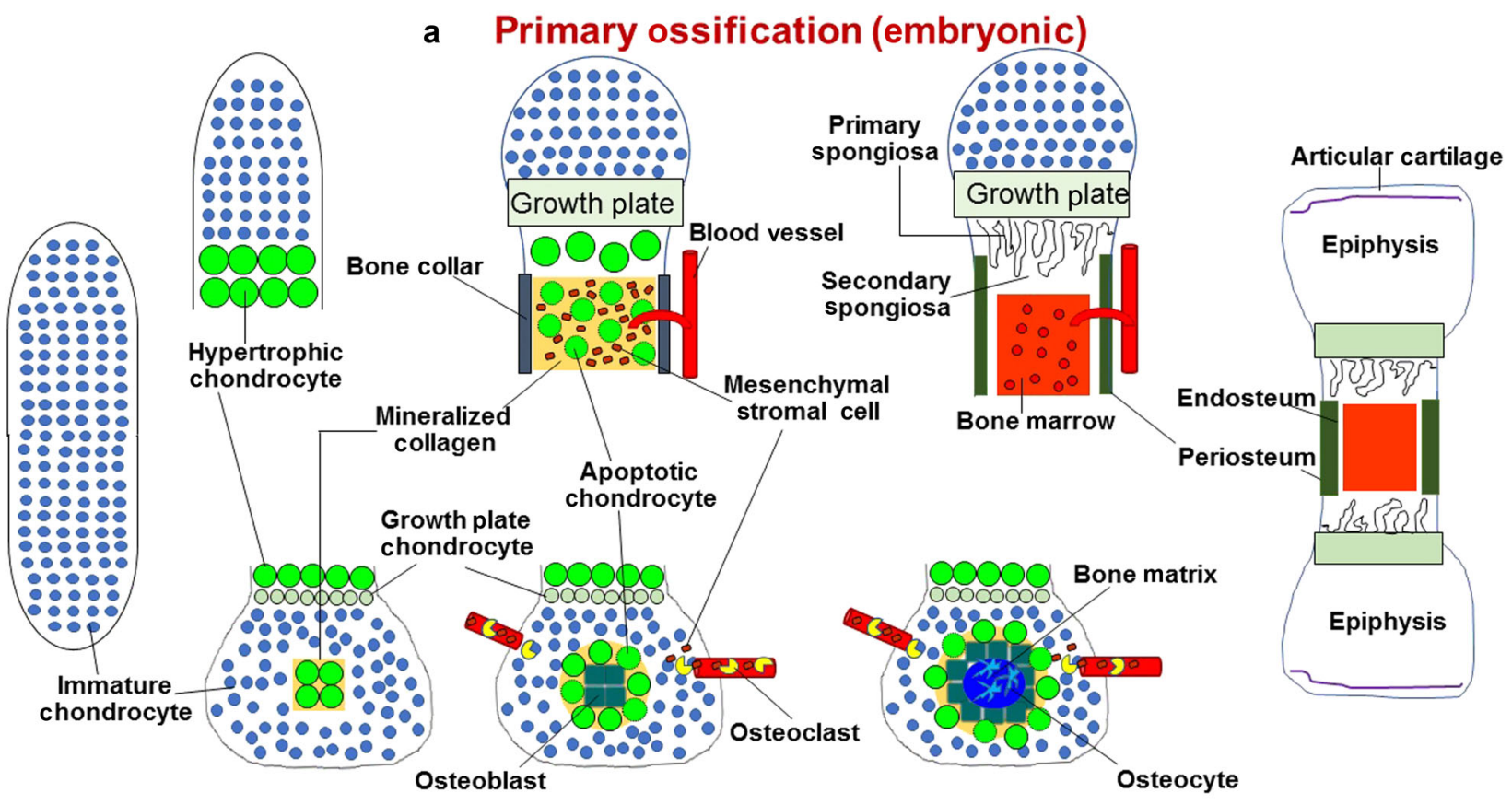

\section{b Secondary ossification (postnatal)}

Fig. 1 Existing paradigm for long bone endochondral ossification. a Primary endochondral ossification begins with the formation of a chondrocyte template during embryogenesis. Chondrocytes undergo hypertrophy beginning from the mid-diaphysis, eventually extending to the epiphyseal poles. Vasculature invades the forming bone, transporting marrow, mesenchymal stromal cells, and osteoclasts. Hypertrophic cells undergo apoptosis, aided by the removal of matrix by osteoclasts. Mesenchymal stromal cells differentiate into osteoblasts and then osteocytes. b Secondary ossification occurs at the epiphysis post-natally in rodents. Immature chondrocytes at the center of the epiphysis become hypertrophic to produce mineralized collagen and eventually undergo apoptosis. Vasculature invades and transports marrow, mesenchymal stromal cells, and osteoclast precursors. Bone formation initiates at the center and extends peripherally.

phosphatase and BMP-2 activation. ${ }^{15}$ In a reversal of roles, rat chondrocytes have been shown, when stimulated by neurogenic growth factors (FGF-2, Neurobasal-A, EGF, and IGF-1), to transdifferentiate into stellate neuronal cells with ablation of $\mathrm{COL} 2$ expression and expression of neuron-specific proteins such as NF200, MAP-2, and $\beta$-III tubulin. ${ }^{16}$ Another case of osteogenic transdifferentiation involves the dedifferentiation of myoblasts via BMP-2 induction of SMAD1, which is mediated by osteoactivin. Osteoactivin, in turn, downregulates myogenic markers and upregulates osteogenic markers, such as RUNX2 and ALP. , 17-20 $^{2}$ Finally, human gingivial fibroblasts have been shown, both in vitro and in vivo to transdifferentiate to osteoblastic lineage cells when treated with 5-aza-2'-deoxycytidine followed by subsequent treatment with BMP2. This was confirmed in vitro by upregulation of Runx2 and Alp expression and in vivo subcutaneous transplantation into mouse, which resulted in increased bone mineral content and bone volume/tissue volume. ${ }^{21}$ The issue of whether the transdifferentiation observed in some of these in vitro studies can be attributed to the resident mesenchymal stem cells present in the cultures used remains to be examined.

Beyond these examples, it is important to note that any process of developing induced pluripotent stem cells from somatic cells is a form of intermediate transdifferentiation. For example, in the famous study by Takahashi and Yamanaka, mouse embryonic fibroblasts were transduced to express what are now known as the Yamanaka factors $(O c t 3 / 4$, Sox2, c-Myc, KIf4) to induce a pluripotent cell intermediate. These cells were then able to form teratomas in cell culture settings and when introduced into an undifferentiated blastocyst, were able to follow normal differentiation programming indistinguishable from the natural pluripotent stem cells. ${ }^{22}$ Transdifferentiation is, therefore, not limited to artificial cell culture settings, but a natural phenomenon.
While there are many examples of transdifferentiation in the literature, this review will focus mainly on recent examples of transdifferentiation relating to the transformation of chondrogenic tissue into osteogenic tissue and the underlying mechanisms.

\section{VASCULAR SOURCE OF OSTEOGENIC PRECURSORS IN THE CANONICAL PATHWAY OF ENDOCHONDRAL OSSIFICATION}

To understand the different mechanisms of chondrocyte-toosteoblast transdifferentiation, it is first important to reference the traditional canonical mechanism of endochondral ossification. $^{23-28}$ Endochondral ossification, in the long bones, is the process of replacing cartilage with bone (Figs. 1 and 2a). This process differs in various bone tissues. In the long bones themselves, there are different temporo-regional mechanisms of endochondral ossification. These processes are referred to as primary endochondral ossification (Fig. 1a) and secondary ossification (Fig. 1b). Primary ossification begins at embryonic day (E) 14.5-15.5 in rodents and encompasses the formation of the periosteum, the bone collar (osteoid layer), and trabeculae which begins at the mid-diaphysis and extends to the growth plates. Mechanistically, the most accepted mechanism for these models begins with the formation of a template of rapidly proliferating immature chondrocytes, which secrete a type 2 collagen (COL2) matrix and make up both the perichondrium and the immature chondrocytes of the diaphysis. This matrix is then degraded when chondrocytes undergo hypertrophic differentiation and secrete enzymes such as matrix metalloprotease 13 (MMP13) and ADAM-TS4. ${ }^{27,29-31}$ Chondrocytes then begin to proceed through apoptosis and the perichondrium becomes periosteum, while the inner layer of the periosteum undergoes intramembranous ossification to form bone collar which encloses 


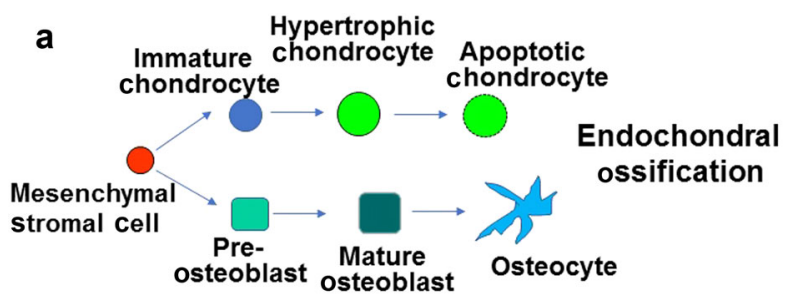

b

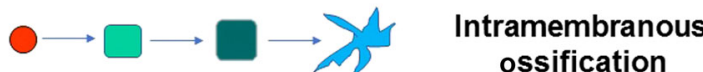

C

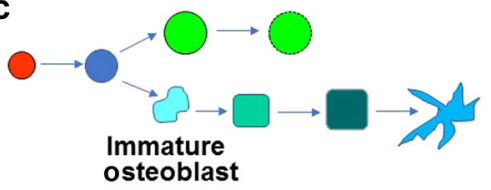

Chondrocyte to osteogenic precursor

d

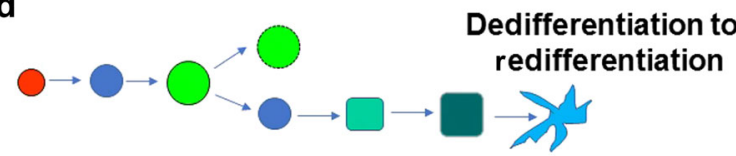

e

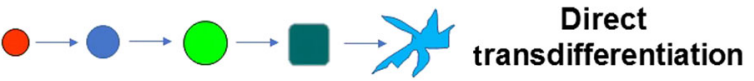

Fig. 2 Models of bone formation. a Endochondral ossification. Mesenchymal stromal cells develop into two different lineages, chondrogenic and osteogenic with no other intermediates. b Intramembranous ossification. Osteoblast development does not require the formation of a chondrocyte template. Mesenchymal stromal cells directly differentiate in an osteogenic lineage. c Chondrocyte to osteogenic precursor. Immature chondrocytes differentiate into an osteogenic precursor population which then differentiate into pre-osteoblasts and osteoblasts. d Dedifferentiation to redifferentiation. Hypertrophic chondrocytes dedifferentiate into immature chondrocytes, which directly differentiate to an osteogenic fate. e Direct transdifferentiation. Hypertrophic chondrocytes directly differentiate to osteoblasts.

the chondrocytes in the center of the bone. Concurrently, the tissue is invaded by incoming vasculature. Osteoclasts from the invading vasculature remove chondrocytes from the template and mesenchymal stromal cells replace apoptosing chondrocytes. ${ }^{27,32}$ Mesenchymal stromal cells then differentiate into osteoblasts to produce type 1 collagen (COL1) and other bone matrix proteins such as bone sialo protein (BSP) and osteocalcin which stimulate bone mineralization. ${ }^{33,34}$ Secondary endochondral ossification occurs in a very similar manner. The principal differences between primary and secondary ossification are the time at which they occur. Secondary ossification initiates at post-natal day (P) 7-8 in rodents in the mid-epiphysis, and then expands peripherally to widen the bone, while primary ossification is involved more in longitudinal growth. ${ }^{26,35}$ Furthermore, secondary ossification largely lacks a periosteal layer and ossification of both ends of the long bone epiphysis may occur at different time points, as the proximal femur epiphysis develops bone much later than the distal femur epiphysis in humans.

As mentioned earlier, endochondral ossification is not the only method by which bone formation occurs. Intramembranous ossification plays an important role in the formation of flat bones in the skull, mandible, and clavicle. ${ }^{36,37}$ More importantly, as it relates to the long bones, intramembranous ossification is responsible for the formation of the subperiosteal surface. Instead of first forming a cartilage intermediate which is then replaced by bone via a tightly coupled process involving chondrocyte, osteoblast, and vascular differentiation, periosteum is formed directly by differentiating mesenchymal cells. These cells then differentiate directly into osteoblasts and secrete COL1 and proteoglycans to form an osteoid matrix, which is then mineralized to form the bone ${ }^{38}$ (Fig. 2b). Mesenchymal stromal cells which are enclosed by the osteoid layer differentiate into osteocytes, including cells of the periosteum. Bone formed via endochondral ossification does not form nodules, and requires a chondrogenic template. Furthermore, intramembranous ossification does not require chondroclasts or osteoclasts for initial remodeling.

Endochondral ossification is also involved in the natural healing of bone fractures. Although fracture healing involves both intramembranous and endochondral ossification, the formation of cartilaginous callus, which later undergoes mineralization and resorption for subsequent replacement with bone, represents the primary method by which fracture healing proceeds. After fracture, the blood clots form a hematoma, which is then stabilized by the surrounding periosteum and other tissues. The inner layer of the periosteum, or inner cambium, produces a mass of chondrocytes to form a template very similar to developmental endochondral ossification. These cells then proceed through normal ossification and form both a hard and soft callus in place of the fracture. ${ }^{39,40}$ It is traditionally accepted that the primary source of osteoblasts during endochondral ossification both during skeletal development and fracture repair is through invading blood vessels that bring in mesenchymal stem cells which proliferate and differentiate to become osteoblasts. However, in contrast to the canonical pathway involving chondrocyte apoptosis and perivascular location as the origin of mesenchymal stem cells, we and others have recently found that chondrocytes undergo transdifferentiation into bone-matrixproducing osteoblasts both during normal endochondral ossification as well as during fracture repair.

The classic model of endochondral ossification emphasizes the apoptotic fate of terminally differentiated chondrocytes which was first suggested by characteristic changes in morphology, and, more recently, by the pattern of DNA fragmentation and other characteristic features of apoptosis. ${ }^{1741-43}$ Based on the findings from several laboratories that the rate of chondrocyte apoptosis increased during fracture healing, it is generally accepted that hypertrophic chondrocytes are programmed to die during the process of endochondral ossification. However, other studies have indicated evidence for transformation of hypertrophic chondrocytes into osteoblasts or other cell types. In this regard, our previous study revealed that Bax deficiency resulted in increased cartilage production that is caused by increased proliferation but without changes in apoptosis. ${ }^{44}$ Furthermore, we and others ${ }^{44-46}$ have found that the change in apoptotic rate of chondrocytes in response to fracture is rather small (approximately 5\%), thus not excluding other fates of hypertrophic chondrocytes such as transdifferentiation into osteoblasts.

\section{CHONDROCYTE-TO-OSTEOBLAST TRANSDIFFERENTIATION} MODELS

As mentioned earlier in this review, the canonical bone development model involves the differentiation of mesenchymal stem cells into a specific cell lineage fate. Typically, depending on the time and signaling, a mesenchymal stromal cell will differentiate through one specific lineage, either chondrogenic, adipogenic, or osteogenic. The end of each will result in terminal differentiation (Fig. 2a). In this model, chondrocytes that hypertrophy will typically eventually apoptose. ${ }^{27,32}$ Beyond this, there are currently three known major models of transdifferentiation of chondrocytes to osteoblasts, and each are mechanistically different and may be specific for different skeletal sites during embryonic and post-natal 
a

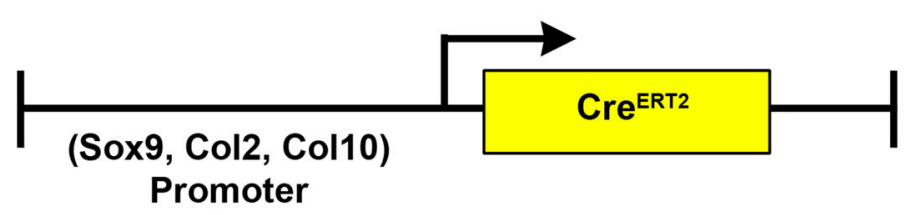

$\mathbf{X}$
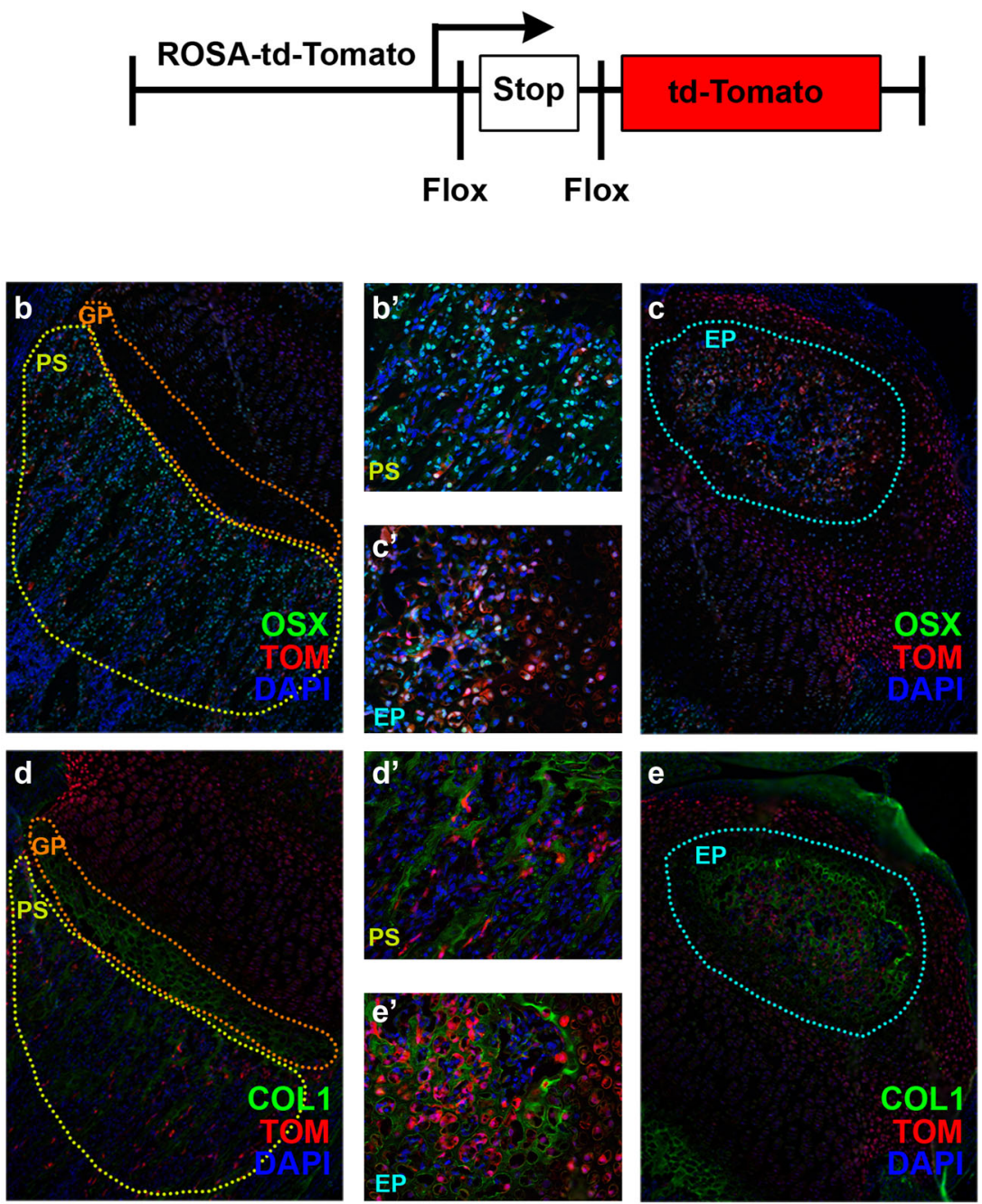

Fig. 3 Lineage tracing of chondrocytes to osteoblasts. a The lineage trace construct consists of a tamoxifen-inducible CRE, which is driven by a promoter of a chondrogenic tissue, such as Sox9, Col2, Col10, or Acan. This in turn excises the stop codon blocking the ROSA-LSL-TdTomato construct. All further cells in the recombined lineage will express td-tomato. b, b' ROSA-LSL-TdTomato floxed mice, positive for Col2-Cre ERT2 $^{\prime}$ were administered with tamoxifen at P3 and mice euthanized at P10. Coexpression of OSX and Tomato-red in cells of the primary spongiosa. c, $\mathbf{c}^{\prime}$ Coexpression of OSX and Tomato-red in cells of the epiphysis. $\mathbf{d}, \mathbf{d}^{\prime}$ Coexpression of COL 1 and Tomato-red in cells of the primary spongiosa. e, e' Coexpression of COL1 and Tomato-red in cells of the epiphysis. COL1, collagen type 1; OSX, osterix; TOM, Tomato-red; DAPI, nuclear stain; PS, primary spongiosa; GP, growth plate; EP, epiphysis.

growth periods. We will differentiate each model by designating them with the method by which the chondrocytes differentiate. The first two models of which imply intermediate transdifferentiation and the final model which implies direct transdifferentiation.

The chondrocyte to osteogenic precursor (OP) model suggests that immature chondrocytes in the growth plate can differentiate into a transient osteogenic precursor in the metaphysis. ${ }^{47}$ During rapid bone growth, this provides a mechanism for generation of both stromal cells and osteoblasts (Fig. 2c). In this model, the transient osteogenic precursors are not thought to self-renew. In addition, this model seems to be specific for metaphyseal growth, much of which occurs embryonically during primary ossification, but can extend into post-natal growth and are separate from adult mesenchymal progenitors. Experimental support for this idea is drawn from fate mapping studies in which cells were lineage traced using a tamoxifen-inducible CRE preceded by promoters for genes expressed by immature chondrocytes such as Col2, aggrecan (Acan), and SRY-Box9 (Sox-9) to induce expression of the 
red fluorescent protein variant TdTomato (Fig. 3a). Pulse chase tamoxifen experiments during embryonic development suggested that cells originating from Osx-CreER proliferate and persist for a brief time in the primary spongiosa and metaphysis, only to disappear, while those originating from Col2-CreER animals continually form bone in the perichondrium, primary spongiosa, and secondary ossification centers, supporting the idea of a transient intermediate. In this study, the authors speculate that the early mesenchymal progenitors expressing chondrocyte markers provide a rapid source of osteoblasts during the rapid phase of bone growth, but are different from adult mesenchymal progenitors. $^{48}$

The dedifferentiation to redifferentiation model provides an alternate view of chondro-osteogenic transdifferentiation. In this model, chondrocytes hypertrophy and either enter apoptosis or dedifferentiate first into immature chondrocytes, and then redifferentiate into osteoblasts and further into osteocytes (Fig. 2d). This model has been described to occur during embryonic and post-natal development, but also during fracture healing. ${ }^{49}$ Specifically, Zhou et al. implied in their experiments that chondrocytes transdifferentiate into osteoblasts and contribute to longitudinal growth in long bones. In this work, the authors used a tamoxifen-inducible Acan-CreERT2 or Col10-Cre (a marker typically used to identify hypertrophic chondrocytes) to drive the expression of a ROSA-LSL-TdTomato marker, this was coupled with a 2.3Col1-GFP reporter to show that, in fact, the osteoblasts lineage arose from the transdifferentiating hypertrophic chondrocytes. Furthermore, Acan-CreERT2; ROSA-LSL-TdTomato cells expressed 2.3Col1-GFP at the ossified fracture calluses at 14 days postsurgery, suggesting that a chondrocyte-to-osteoblast transdifferentiation mechanism is at least somewhat involved in fracture healing. This mechanism was confirmed by another group using both Acan-CreERT2 and Col2-CreERT2. ${ }^{50}$ Additionally, Hu et al. reported the expression of stem-like markers (OCT4, NANOG, SOX2) in chondrocytes located in the fracture callus, suggesting the possibility of dedifferentiation. ${ }^{50}$ In a subsequent study, the model of dedifferentiating chondrocytes was further proposed using a tamoxifen-inducible Col10-ERT2 to drive YFP expression, and then colabeled with markers typically found in osteogenic cells (COL1, OSX) during mandibular growth, further solidifying evidence that osteoblasts are able to arise from hypertrophic chondrocyte lineages. ${ }^{51}$ In another study, Yang et al. ${ }^{52}$ demonstrated using Col10-CreERT2 that hypertrophic chondrocytes may become Col1a1-expressing osteoblasts and sclerostin-expressing osteocytes in prenatal and post-natal bones and during bone injury repair. While these studies certainly suggest the possibility of hypertrophic chondrocytes dedifferentiating first before differentiating into osteogenic tissue, this hypothesis has been left open for interpretation.

Differentiation of stem cells into specialized cells requires an upregulation of genes involved in the creation of a specific cell phenotype and suppression of genes responsible for cell stemness. $^{53}$ In a recent study, Kang et al. $^{54}$ evaluated the expression of 11 stemness genes during in vitro differentiation of induced pluripotent stem cells into mesenchymal stem cells and tri-lineage (osteoblast, chondrocyte, and adipocyte) differentiated cells. They found that while all stemness genes were expressed in induced pluripotent stem cells, most of the stemness genes except KIf4 and C-Myc were not expressed in the tri-lineage differentiated cells. These findings are in favor of use of stemness gene expression marker to identify osteogenic stem cells. However, the issue of whether the identified stem cell marker gene expression is in dedifferentiated hypertrophic chondrocytes per se and not in the contaminating stem cells present in tissue preparations examined needs to be carefully investigated before concluding the involvement of an intermediate dedifferentiation step during osteogenic differentiation of hypertrophic chondrocytes.
Finally, the direct transdifferentiation model suggests that direct transdifferentiation is the method by which post-natal secondary ossification occurs. The direct transdifferentiation model suggests that chondrocytes will mature and hypertrophy, and that they do not apoptose, but instead differentiate directly into osteoblasts and subsequently into osteocytes (Fig. 2e). In a recently published study, we administered tamoxifen at post-natal (P) day 3 to Col2CreERT2 animals to activate the tdTomato reporter in early immature chondrocytes. These cells then proceed to present themselves in newly formed bone of the epiphysis by co-localizing with bone markers such as OSX, BSPII, ALP, DMP1, OCN, and COL1. Prior to this, these same cells will express markers typically seen in hypertrophic chondrocytes such as COL10 and MMP13. The tdTomato marked cells have been found to be embedded in bone matrix as osteoblasts and osteocytes after several days (Fig. 3b-e') or weeks ${ }^{4}$ of post-natal development. This process all initiates before vascular invasion of the epiphysis reaches the region of secondary ossification. Furthermore, there is no observed increase in proliferation or apoptosis observed in the epiphyseal hypertrophic chondrocytes. Coupled with the co-localization data with bone markers, transdifferentiation seems to be an important mechanism for early post-natal bone formation. Even a study as early as 1992 in chick embryos suggested that hypertrophic chondrocytes have the capability to transdifferentiate into bonematrix-forming cells. ${ }^{55}$ Consistent with this data, we demonstrated that treatment of ATDC5 chondrocytes with thyroid hormone increased the expression levels of osteoblast differentiation markers and bone nodule formation in vitro, thus providing evidence for chondro-osteoblast transdifferentiation. ${ }^{56}$ Based on these in vitro findings and our in vivo findings that hypertrophic chondrocytes express markers of osteoblasts, our model of direct transdifferentiation also suggests that hypertrophic chondrocytes may, in fact, be a misnomer, and that they are in fact preosteoblasts. This model is also supported by experiments using a Col10-CreERT2 to drive fluorescent marker lineage trace of hypertrophic cells suggested that not only did the cells of the growth plate contribute to the primary spongiosa, but that these cells also seemed to directly transdifferentiate. ${ }^{57} \mathrm{~A}$ possible indicator of this is embodied by the expression of OSX, a marker for early osteoblasts that often manifests in late stage hypertrophic chondrocytes. Moreover, this model suggests that direct transdifferentiation is the most likely mechanism for the formation of epiphyseal osteoblasts as the process of dedifferentiation and redifferentiation would not occur quickly enough in the span of time in which the bone appears immediately after the formation of the hypertrophic chondrocytes in the epiphysis. ${ }^{4,56}$ Direct transdifferentiation is not unique to secondary ossification as it probably also occurs during primary ossification as well as during fracture healing.

In a recent study, Sakagami et al. $^{58}$ have evaluated if Col2a1expressing cells contributed various models of ossification occurring during the craniofacial skeletal complex by an in vivo cell mapping technique utilizing Col1a1(2.3 kb)-GFP and Col2a1Cre:ROSA-LSL-tdTomato mice. They found that Col2a1-Cre, as expected, consistently marked most skeletal cells in the bones of cranial base, which primarily form by endochondral ossification route. However, virtually all Cola1-GFP ${ }^{+}$osteoblasts near the suture were green, suggesting that they were not derived from Col2a1-Cre-marked cells. In contrast, many osteoblasts in the inner aspect of the calvaria were marked with Col2a1$\mathrm{Cre}$, thus suggesting that mechanisms of craniofacial bone formation may be complex and utilize both Col2a1 positive and negative early progenitors of the skeletal lineage. Thus, while these and other studies raise the possibility that the early progenitors expressing Col2a1 represent common precursors and could become osteoblasts or chondrocytes, in the context of this review, transdifferentiation refers to the conversion of fully differentiated hypertrophic chondrocytes 
into osteoblasts rather than undergoing their preprogrammed cell death.

\section{SIGNALS THAT CONTROL CHONDROCYTE-TO-OSTEOBLAST TRANSDIFFERENTIATION}

In order to properly investigate the process of transdifferentiation, it is important to define the signals involved in specifying the phenotype of varying cell types to discern how much of each of the signaling processes are conserved in different tissues. While signaling may play a role in causing cell differentiation, at times, the lack of a signal also plays a role. In the example of hindgut to midgut transition in Drosophila, the adult hindgut progenitors rely on wingless (WG) signaling (a homolog of WNT) to keep them in an undifferentiated state. Once cells of the anterior hindgut proliferation zone (HPZ) migrate anteriorly, they eventually cross a threshold that prevents cell renewal, and instead are exposed to other molecular signals that induce this transition. Moreover, cells of the HPZ express GATAe which is required to induce migration and subsequent loss of hindgut fate markers. Additionally, in $\mathrm{Wg}$ overexpressing animals, this event does not occur, and in fact, the cells do not even migrate. ${ }^{9}$ Wnt also plays a role in the induction of other cell types into the osteogenic lineage, as well as, inhibiting osteogenic cells from transdifferentiating into chondrogenic or adipogenic lineages. ${ }^{59-65}$ This is much different than the specific signals noted here for chondrocyte-to-osteoblast transdifferentiation, but other examples, such as the formation of iPSCs, require factors like Oct4 and Sox2 expression, which was also one of the factors involved in the dedifferentiation described in a paper using the DR model of chondrocyte transdifferentiation. ${ }^{22,50}$

While it is not known exactly what causes the transdifferentiation of chondrocytes into osteoblasts, it seems that the expression of some factors is necessary for that transition. Factors such as IGF1 are known to be important in regulating chondrocyte and osteoblasts proliferation and maintenance. ${ }^{6-68}$ IGF-1 also regulates osteoblast differentiation from mesenchymal stromal cells through mTOR. ${ }^{69}$ Knockouts of IGF-1 also reduce the anabolic effects of important osteogenic hormones such as parathyroid hormone (PTH) ${ }^{70,71}$ Perhaps it has a role to play in chondrocyteto-osteoblast transdifferentiation, but that remains to be seen. Teriparatide, a recombinant of PTH approved for clinical use, has been shown to increase proliferation and differentiation of Sox9cre chondrogenic precursors to osteogenic fates, that it functions through the PTH receptor, and that upon withdrawal of teriparatide treatment, these precursors do not undergo chondrogenic differentiation, but instead develop into an adipogenic lineage. ${ }^{72}$ The importance of WNT signaling in endochondral ossification is well established by studies on disruption of $\beta$ catenin specifically in chondrocytes. ${ }^{60,63,73}$ In terms of mechanisms for regulation of IGF-I and WNT signaling during endochondral ossification, it is known that thyroid hormone levels begin to spike at late pre-natal and early post-natal growth, which are key boneforming time points. ${ }^{56,74}$ This rise in thyroid hormone levels during the second week of post-natal life is indispensable for the IGF-I and $\beta$-catenin expression in the epiphysis. ${ }^{4,56}$ Further, the expression of Runx2 and its downstream effector Osx are essential for ossification to occur in the epiphysis and trabecular bone. ${ }^{75}$ Osx is also an important regulator in cementogenesis, ${ }^{76}$ a process that is very similar to that of bone formation. ${ }^{77,78}$ In the case of cementogenesis, Osx is a major downstream regulator of the Wnt/ $\beta$-catenin signal transduction pathway and is absolutely necessary for this process to proceed. ${ }^{76,79}$ Absence of circulating thyroid hormone which functions through modulation of Osx and Indian hedgehog $(I h h)$ expression has been observed to prevent bone formation in the epiphysis. In our recent work, we have shown that thyroid hormone-deficient animals could form prehypertrophic chondrocytes, but bone formation was halted during normal ossification windows, and in later stages was dysfunctional. Much of this was related to the inability of chondrocytes to transdifferentiate into osteoblasts. ${ }^{56}$ An important role for calcium and its putative receptor, calcium sensing receptor, in transdifferentiation can also be speculated based on the established effects of extracellular calcium in promoting the differentiation of chondrocytes and osteoblasts. ${ }^{80-82}$

In an example of lens regeneration in newts using cells from other tissues, the transdifferentiation of the epithelial cells of the dorsal iris into lens cells was found to increase expression of both Sonic hedgehog (Shh) and $\mathrm{lhh} .^{83} \mathrm{IHH}$ has been shown to be an important factor for differentiation of immature chondrocytes into a hypertrophic state by regulating levels of parathyroid hormonerelated protein (PTHrP), which keeps chondrocytes in an immature proliferative state. $^{64,84} \mathrm{IHH}$ also activates GLI2, which activates osteogenic differentiation. ${ }^{85-92}$ This is mediated in part through the GLI2 activation of Runx2 and Osx expression. ${ }^{56,91}$ Once the correct balance of IHH expression is achieved, chondrocytes and mesenchymal cells can differentiate. As suggested in Aghajanian et al., IHH-mediated Gli2 expression is a very likely mediator of osteogenic transdifferentiation of chondrocytes. ${ }^{4}$ This point is further corroborated in a study that suggests IHH and GLI2 upregulate Runx2, Alp, and Ocn expression along with increased bone mineralization. Moreover, this effect was not observed by Gli3 overexpression and Gli2 dominant negative animals inhibited the $\mathrm{IHH}$ dependent effect on bone formation. ${ }^{91}$ Investigators in another study observed the coexpression of both bone (Osx, Coll, Runx2) and cartilage (Sox9, Col2, Col10) genes during jawbone repair in zebrafish, suggesting a transdifferentiation program. Moreover, in $\mathrm{Ih}^{-/-}$zebrafish, callus formation after fracture is greatly reduced, as is bone mineralization and periosteal bone formation, while chondrocyte proliferation in the fracture region remains unaffected. ${ }^{93}$ Finally, Ihh expression has been shown to increase under the influence of thyroid hormone expression (a known inducer of bone formation), and specifically through thyroid hormone receptor $\beta 1$ (TR $\beta 1)$. Direct interaction between Ihh and TR $\beta 1$ was confirmed via the presence of a thyroid hormone response element (TRE) in the promoter of the Ihh gene. $^{94}$

Runx2, a known factor in osteogenic differentiation, was thought to be important in the transdifferentiation process in the chondrocyte to osteogenic precursor model. ${ }^{47}$ The role of Runx2 as a master regulator of osteogenic fate has been further implicated in the process by its important role in the transdifferentiation of adipocytes, ${ }^{95}$ primary skeletal myoblasts, ${ }^{96}$ odontoblasts, ${ }^{97}$ and vascular smooth muscle cells ${ }^{59}$ to osteogenic cell types. Runx2 activation usually comes in conjunction with the usual secreted factors such as bone morphogenic proteins (BMPs) or WNTs. An example of this is the transdifferentiation of myoblasts into osteoblasts via induction by both RUNX2 and BMPs. Naturally, BMPs have the ability to not only upregulate the expression of osteogenic factors, but also downregulate expression of myogenic factors. ${ }^{18}$ As it pertains to transdifferentiation, Cho et al. indicated that BMP2 is an important regulator of osteogenic transdifferentiation from gingivial fibroblasts. ${ }^{21}$ In our studies, we found that thyroid hormone upregulated expression levels of many growth factors (IHH, BMP, IGF-I, and RANKL) as well as transcription factors (RUNX2, OSX, $\beta$-CATENIN) during epiphyseal bone formation, thus suggesting involvement of multiple signaling pathways in driving the chondrocyte-osteoblast transdifferentiation process (Fig. 4). Additional in vivo studies using appropriate animal models are needed to determine the relative contribution of the various signaling pathways identified in in vitro studies in regulating chondrocyte-to-osteoblast transdifferentiation.

\section{Implications for clinical practice}

The clinical relevance of understanding the potential contribution of the chondrocyte-to-osteoblast differentiation route to endochondral ossification and the molecular mechanisms that 

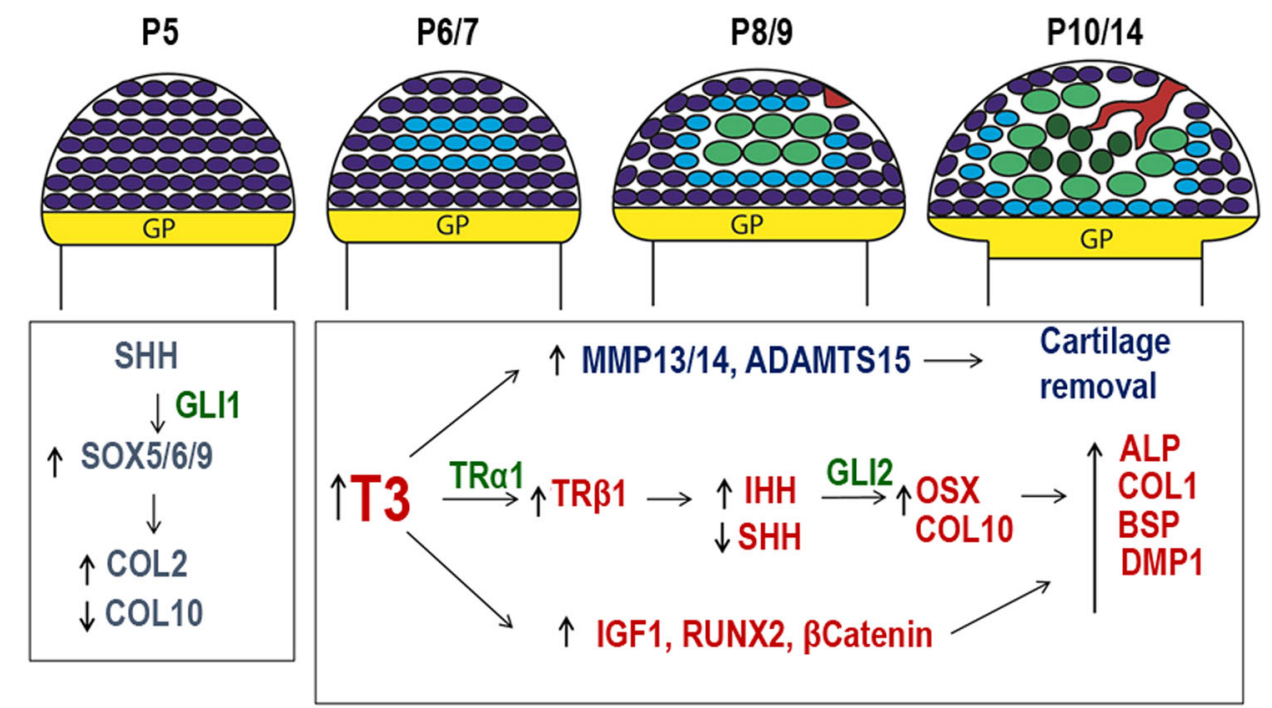

Immature CC

Pre-hypertrophic CC

Hypertrophic CC

Osteoblast

Fig. 4 Proposed model for thyroid hormone-mediated early post-natal development of the secondary ossification center (SOC). During embryonic and early post-natal development when thyroid hormone (TH) levels are low, epiphyseal chondrocytes express elevated levels of $\mathrm{SHH}$, which acts through GLI1 to maintain these cells in proliferative immature state by activating Sox5/6/9 transcriptional activity. At P6/P7, rise in TH increases TR 31 expression and thereby IHH expression. IHH acts through GLI2 to decrease SOX9 and COL2 expression, while MMP13 and ADAMTS5 expression increases to deplete the COL2 matrix. Pre-hypertrophic chondrocyte (CC)s begin to express COL10 and OSX in the P8/P9 period. These in turn activate DMP1 and ALP in the SOC, meanwhile blood vessels invade from the periphery of the articular CCs. Text and image obtained from ref. ${ }^{4}$

contribute to the switch from chondroblastic to osteoblastic cellular machinery are as follows. 1) The current therapies for nonunion fractures involve strategies that utilize mesenchymal stem cells-derived from patients and/or growth factors to promote direct bone formation at the fracture site. However, these strategies have not been proven to be effective and may be cost prohibitive. Since chondrocyte proliferation and differentiation predominate early during the fracture repair process, it may be more prudent to expand the existing chondrocytes and convert them to osteoblasts capable of performing all of the functions required to resorb cartilage and produce bone as performed by the epiphyseal chondrocytes in secondary ossification centers when thyroid hormone levels are high. Our understanding of the thyroid hormone-induced molecular events that lead to time and space-specific conversion of chondrocytes into osteoblasts could lead to potential breakthroughs in terms of identifying novel therapeutic strategies to heal non-union fractures. 2) The default route of chondrocyte differentiation is predicted to be terminal differentiation leading to bone formation. ${ }^{98,99}$ In the articular cartilage, this default route is somehow blocked to obtain permanent articular cartilage. During mechanical injury, inflammation, or aging, the signals that contribute to this blockade are dysregulated leading to a loss of undifferentiated articular chondrocyte progenitors, thus contributing to the pathogenesis of osteoarthritis. Future understanding of the mechanisms involved in temporal and spatial control of chondrocyte to osteoblast differentiation events during endochondral ossification could lead to the development of strategies to manipulate signals that control these events for a therapeutic benefit in the treatment of joint injury and disease. 3) The current anabolic therapies for osteoporosis are based on promotion of osteoblast functions. If the prediction that chondrocytes contribute to an important source of osteoblasts and bone formation processes during both growth and remodeling turn out to be true, then studying the mechanisms that contribute to chondrocyte-to-osteoblast transdifferentiation will provide exciting new strategic approaches to develop anabolic therapies for osteoporosis and other bone wasting diseases.

\section{CONCLUSIONS}

While endochondral ossification seems to proceed using the longknown paradigm, it seems that a transdifferentiation mechanism may be working in conjunction with canonical endochondral ossification to promote bone health. Indeed, it is possible that this process may manifest itself using all the different models mentioned in this review, and that each model has a tempororegional preference. Further, the redundancy observed with transdifferentiation may account for faster tissue regeneration, explosive bone growth, and secondary ossification prior to vascularization of the tissue. As innovative studies become more prevalent in the literature, the contribution of chondrogenic tissue to new bone may provide new avenues for therapeutics for various osteodegenerative diseases and fracture healing methodologies.

\section{FUTURE DIRECTIONS}

The research on transdifferentiation is still in a rudimentary stage and poses a number of unresolved questions which include: (1) Does chondrocyte-to-osteoblast transdifferentiation contribute to the pathogenesis of diseases such as osteoarthritis and heterotopic ossification? (2) Can targeted therapies which promote chondrocyte-to-osteoblast transdifferentiation be developed to promote healing of large skeletal defects and nonunion fractures? (3) Does thyroid hormone regulation of post-natal development in other tissues involve transdifferentiation? (4) Does transdifferentiation have a broader implication in fields such as cancer, cardiovascular diseases, and diabetes? and (5) Can modulation of transcription factors involved in transdifferentiation be used as an effective framework for direct reprograming between cell types? 


\section{ACKNOWLEDGEMENTS}

This work was supported by funding from the National Institutes of Arthritis and Musculoskeletal Diseases RO1 grant (AR048139) and Veterans Administration BLR\&D merit review grant (101-BX-001396) to S.M. S.M. is a recipient of a Senior Research Career Scientist Award from the Department of Veterans Affairs. The authors thank Dr. Donna Strong for proof reading the manuscript. Figure 4 was obtained from Aghajanian et al. ${ }^{4}$ and is unmodified. A copy of the creative commons license for this figure can be found here: https://creativecommons.org/licenses/by/4.0/legalcode.

\section{ADDITIONAL INFORMATION}

Conflict of interest: The authors declare that they have no conflict of interest.

\section{REFERENCES}

1. Chen, Q. et al. Fate decision of mesenchymal stem cells: adipocytes or osteoblasts? Cell Death Differ. 23, 1128-1139 (2016).

2. Doseff, A. I. Apoptosis: the sculptor of development. Stem Cells Dev. 13, 473-483 (2004).

3. Kami, D. \& Gojo, S. Tuning cell fate: from insights to vertebrate regeneration. Organogenesis 10, 231-240 (2014).

4. Aghajanian, P., Xing, W., Cheng, S. \& Mohan, S. Epiphyseal bone formation occurs via thyroid hormone regulation of chondrocyte to osteoblast transdifferentiation. Sci. Rep. 7, 10432 (2017).

5. Cieslar-Pobuda, A. et al. Transdifferentiation and reprogramming: overview of the processes, their similarities and differences. Biochim. Biophys. Acta 1864, 1359-1369 (2017).

6. Coll-Bonfill, N., Musri, M. M., Ivo, V., Barbera, J. A. \& Tura-Ceide, O. Transdifferentiation of endothelial cells to smooth muscle cells play an important role in vascular remodelling. Am. J. Stem Cells 4, 13-21 (2015).

7. Frasch, M. Dedifferentiation, redifferentiation, and transdifferentiation of striated muscles during regeneration and development. Curr. Top. Dev. Biol. 116, 331-355 (2016).

8. Lin, D. P. L., Carnagarin, R., Dharmarajan, A. \& Dass, C. R. Transdifferentiation of myoblasts into osteoblasts - possible use for bone therapy. J. Pharm. Pharmacol. 69, 1661-1671 (2017)

9. Takashima, S., Paul, M., Aghajanian, P., Younossi-Hartenstein, A. \& Hartenstein, V. Migration of Drosophila intestinal stem cells across organ boundaries. Development 140, 1903-1911 (2013).

10. Zhang, R. et al. In vivo cardiac reprogramming contributes to zebrafish heart regeneration. Nature 498, 497-501 (2013).

11. Freeman, G. Lens regeneration from the cornea in Xenopus laevis. J. Exp. Zool. 154, 39-65 (1963).

12. Day, R. C. \& Beck, C. W. Transdifferentiation from cornea to lens in Xenopus laevis depends on BMP signalling and involves upregulation of Wnt signalling. BMC Dev. Biol. 11, 54 (2011).

13. Naitoh, $\mathrm{H}$. et al. Upregulation of matrix metalloproteinase triggers transdifferentiation of retinal pigmented epithelial cells in Xenopus laevis: a link between inflammatory response and regeneration. Dev. Neurobiol. 77, 1086-1100 (2017).

14. Bobryshev, Y. V. Transdifferentiation of smooth muscle cells into chondrocytes in atherosclerotic arteries in situ: implications for diffuse intimal calcification. J. Pathol. 205, 641-650 (2005).

15. Fakhry, M. et al. TNAP stimulates vascular smooth muscle cell trans-differentiation into chondrocytes through calcium deposition and BMP-2 activation: possible implication in atherosclerotic plaque stability. Biochim. Biophys. Acta 1863, 643-653 (2017).

16. Greene, C. A., Green, C. R. \& Sherwin, T. Transdifferentiation of chondrocytes into neuron-like cells induced by neuronal lineage specifying growth factors. Cell Biol. Int. 39, 185-191 (2015).

17. Adams, C. S. \& Shapiro, I. M. The fate of the terminally differentiated chondrocyte: evidence for microenvironmental regulation of chondrocyte apoptosis. Crit. Rev. Oral. Biol. Med. 13, 465-473 (2002).

18. Hayashi, M. et al. Pitx2 prevents osteoblastic transdifferentiation of myoblasts by bone morphogenetic proteins. J. Biol. Chem. 283, 565-571 (2008).

19. Rauch, C., Brunet, A. C., Deleule, J. \& Farge, E. C2C12 myoblast/osteoblast transdifferentiation steps enhanced by epigenetic inhibition of BMP2 endocytosis. Am. J. Physiol. Cell Physiol. 283, C235-C243 (2002).

20. Sondag, G. R. et al. Osteoactivin induces transdifferentiation of $\mathrm{C} 2 \mathrm{C} 12$ myoblasts into osteoblasts. J. Cell. Physiol. 229, 955-966 (2014).

21. Cho, Y. et al. Direct gingival fibroblast/osteoblast transdifferentiation via epigenetics. J. Dent. Res. 96, 555-561 (2017).

22. Takahashi, K. \& Yamanaka, S. Induction of pluripotent stem cells from mouse embryonic and adult fibroblast cultures by defined factors. Cell 126, 663-676 (2006).
23. Kronenberg, H. M. Developmental regulation of the growth plate. Nature 423, 332-336 (2003)

24. Lefebvre, V. \& Bhattaram, P. Vertebrate skeletogenesis. Curr. Top. Dev. Biol. 90, 291-317 (2010)

25. Lefebvre, V. \& Smits, P. Transcriptional control of chondrocyte fate and differentiation. Birth Defects Res. C Embryo Today 75, 200-212 (2005).

26. Long, F. \& Ornitz, D. M. Development of the endochondral skeleton. Cold Spring Harb. Perspect. Biol. 5, a008334 (2013).

27. Mackie, E. J., Ahmed, Y. A., Tatarczuch, L., Chen, K. S. \& Mirams, M. Endochondral ossification: how cartilage is converted into bone in the developing skeleton. Int. J. Biochem. Cell Biol. 40, 46-62 (2008).

28. Mackie, E. J., Tatarczuch, L. \& Mirams, M. The skeleton: a multi-functional complex organ: the growth plate chondrocyte and endochondral ossification. J. Endocrinol. 211, 109-121 (2011).

29. Karsenty, G. in The Skeletal System (ed Pourquie, O.) 205-218 (Cold Spring Harbor Laboratory Press, Cold Spring Harbor, NY, 2009).

30. Karsenty, G., Kronenberg, H. M. \& Settembre, C. Genetic control of bone formation. Annu. Rev. Cell Dev. Biol. 25, 629-648 (2009).

31. $\mathrm{Wu}, \mathrm{W}$. et al. Cartilage matrix resorption in skeletogenesis. Novartis Found. Symp. 232, 158-166 (2001).

32. Sivaraj, K. K. \& Adams, R. H. Blood vessel formation and function in bone. Development 143, 2706-2715 (2016).

33. Ducy, P., Zhang, R., Geoffroy, V., Ridall, A. L. \& Karsenty, G. Osf2/Cbfa1: a transcriptional activator of osteoblast differentiation. Cell 89, 747-754 (1997).

34. Florencio-Silva, R., Sasso, G. R., Sasso-Cerri, E., Simoes, M. J. \& Cerri, P. S. Biology of bone tissue: structure, function, and factors that influence bone cells. BioMed. Res. Int. 2015, 421746 (2015).

35. Iwata, T. et al. A neonatal lethal mutation in FGFR3 uncouples proliferation and differentiation of growth plate chondrocytes in embryos. Hum. Mol. Genet. 9, 1603-1613 (2000).

36. Baron, R. in Anatomy and Ultrastructure of Bone - Histogenesis, Growth and Remodeling (eds De Groot, L. J. et al.) (Endotext, South Dartmouth, MA, USA, 2000).

37. Berendsen, A. D. \& Olsen, B. R. Bone development. Bone 80, 14-18 (2015).

38. Gibert, S. F. (ed) Osteogenesis: The Development of Bones (Sinauer Associates, Sunderland, MA, USA, 2000).

39. Dwek, J. R. The periosteum: what is it, where is it, and what mimics it in its absence? Skeletal Radiol. 39, 319-323 (2010).

40. Shapiro, F. Bone development and its relation to fracture repair. The role of mesenchymal osteoblasts and surface osteoblasts. Eur. Cells Mater. 15, 53-76 (2008).

41. Gibson, G. Active role of chondrocyte apoptosis in endochondral ossification. Microsc. Res. Tech. 43, 191-204 (1998).

42. Shapiro, I. M., Adams, C. S., Freeman, T. \& Srinivas, V. Fate of the hypertrophic chondrocyte: microenvironmental perspectives on apoptosis and survival in the epiphyseal growth plate. Birth Defects Res. C Embryo Today 75, 330-339 (2005).

43. Tsang, K. Y., Chan, D. \& Cheah, K. S. Fate of growth plate hypertrophic chondrocytes: death or lineage extension? Dev. Growth Differ. 57, 179-192 (2015).

44. Rundle, C. H. et al. Bax deficiency in mice increases cartilage production during fracture repair through a mechanism involving increased chondrocyte proliferation without changes in apoptosis. Bone 43, 880-888 (2008).

45. Gaber, S. et al. Chondrocyte apoptosis enhanced at the growth plate: a physeal response to a diaphyseal fracture. Cell Tissue Res. 335, 539-549 (2009).

46. Lee, F. Y., Choi, Y. W., Behrens, F. F., DeFouw, D. O. \& Einhorn, T. A. Programmed removal of chondrocytes during endochondral fracture healing. J. Orthop. Res. 16, 144-150 (1998).

47. Ono, N., Ono, W., Nagasawa, T. \& Kronenberg, H. M. A subset of chondrogenic cells provides early mesenchymal progenitors in growing bones. Nat. Cell Biol. 16, 1157-1167 (2014).

48. Zhou, B. O., Yue, R., Murphy, M. M., Peyer, J. G. \& Morrison, S. J. Leptin-receptorexpressing mesenchymal stromal cells represent the main source of bone formed by adult bone marrow. Cell Stem Cell 15, 154-168 (2014).

49. Zhou, X. et al. Chondrocytes transdifferentiate into osteoblasts in endochondral bone during development, postnatal growth and fracture healing in mice. PLOS Genet. 10, e1004820 (2014).

50. Hu, D. P. et al. Cartilage to bone transformation during fracture healing is coordinated by the invading vasculature and induction of the core pluripotency genes. Development 144, 221-234 (2017).

51. Park, J. et al. Dual pathways to endochondral osteoblasts: a novel chondrocytederived osteoprogenitor cell identified in hypertrophic cartilage. Biol. Open 4, 608-621 (2015).

52. Yang, L., Tsang, K. Y., Tang, H. C., Chan, D. \& Cheah, K. S. Hypertrophic chondrocytes can become osteoblasts and osteocytes in endochondral bone formation. Proc. Natl Acad. Sci. USA 111, 12097-12102 (2014). 
53. Eslaminejad, M. B., Fani, N. \& Shahhoseini, M. Epigenetic regulation of osteogenic and chondrogenic differentiation of mesenchymal stem cells in culture. Cell J. 15, 1-10 (2013).

54. Kang, R. et al. Mesenchymal stem cells derived from human induced pluripotent stem cells retain adequate osteogenicity and chondrogenicity but less adipogenicity. Stem Cell Res. Ther. 6, 144 (2015).

55. Roach, H. I. Trans-differentiation of hypertrophic chondrocytes into cells capable of producing a mineralized bone matrix. Bone Miner. 19, 1-20 (1992).

56. Xing, W., Cheng, S., Wergedal, J. \& Mohan, S. Epiphyseal chondrocyte secondary ossification centers require thyroid hormone activation of Indian hedgehog and osterix signaling. J. Bone Miner. Res. 29, 2262-2275 (2014).

57. Jing, Y. et al. Chondrocytes directly transform into bone cells in mandibular condyle growth. J. Dent. Res. 94, 1668-1675 (2015).

58. Sakagami, N., Ono, W. \& Ono, N. Diverse contribution of Col2a1-expressing cells to the craniofacial skeletal cell lineages. Orthod. Craniofac. Res. 20(Suppl. 1), 44-49 (2017).

59. Cai, T. et al. WNT/beta-catenin signaling promotes VSMCs to osteogenic transdifferentiation and calcification through directly modulating Runx2 gene expression. Exp. Cell Res. 345, 206-217 (2016).

60. Chen, M. et al. Inhibition of beta-catenin signaling causes defects in postnatal cartilage development. J. Cell Sci. 121(Pt 9), 1455-1465 (2008).

61. Dao, D. Y. et al. Cartilage-specific beta-catenin signaling regulates chondrocyte maturation, generation of ossification centers, and perichondrial bone formation during skeletal development. J. Bone Miner. Res. 27, 1680-1694 (2012).

62. Gao, B. et al. Dose-dependent effect of estrogen suppresses the osteo-adipogenic transdifferentiation of osteoblasts via canonical Wnt signaling pathway. PLOS ONE 9, e99137 (2014).

63. Golovchenko, S. et al. Deletion of beta catenin in hypertrophic growth plate chondrocytes impairs trabecular bone formation. Bone 55, 102-112 (2013).

64. Guo, X., Mak, K. K., Taketo, M. M. \& Yang, Y. The Wnt/beta-catenin pathway interacts differentially with PTHrP signaling to control chondrocyte hypertrophy and final maturation. PLOS ONE 4, e6067 (2009).

65. Hill, T. P., Spater, D., Taketo, M. M., Birchmeier, W. \& Hartmann, C. Canonical Wnt/ beta-catenin signaling prevents osteoblasts from differentiating into chondrocytes. Dev. Cell 8, 727-738 (2005).

66. Clemens, T. L. \& Chernausek, S. D. Genetic strategies for elucidating insulin-like growth factor action in bone. Growth Horm. IGF Res. 14, 195-199 (2004).

67. Mohan, S. \& Kesavan, C. Role of insulin-like growth factor-1 in the regulation of skeletal growth. Curr. Osteoporos. Rep. 10, 178-186 (2012).

68. Nilsson, O., Marino, R., De Luca, F., Phillip, M. \& Baron, J. Endocrine regulation of the growth plate. Horm. Res. 64, 157-165 (2005).

69. Xian, L. et al. Matrix IGF-1 maintains bone mass by activation of mTOR in mesenchymal stem cells. Nat. Med. 18, 1095-1101 (2012).

70. Bikle, D. D. et al. Insulin-like growth factor I is required for the anabolic actions of parathyroid hormone on mouse bone. J. Bone Miner. Res. 17, 1570-1578 (2002).

71. Miyakoshi, N., Kasukawa, Y., Linkhart, T. A., Baylink, D. J. \& Mohan, S. Evidence that anabolic effects of PTH on bone require IGF-I in growing mice. Endocrinology 142, 4349-4356 (2001).

72. Balani, D. H., Ono, N. \& Kronenberg, H. M. Parathyroid hormone regulates fates of murine osteoblast precursors in vivo. J. Clin. Invest. 127, 3327-3338 (2017).

73. Houben, A. et al. beta-catenin activity in late hypertrophic chondrocytes locally orchestrates osteoblastogenesis and osteoclastogenesis. Development 143 3826-3838 (2016).

74. Xing, W. et al. Genetic evidence that thyroid hormone is indispensable for prepubertal insulin-like growth factor-I expression and bone acquisition in mice. $J$. Bone Miner. Res. 27, 1067-1079 (2012).

75. Nakashima, K. et al. The novel zinc finger-containing transcription factor osterix is required for osteoblast differentiation and bone formation. Cell 108, 17-29 (2002).

76. Choi, $\mathrm{H}$. et al. A reciprocal interaction between beta-catenin and osterix in cementogenesis. Sci. Rep. 7, 8160 (2017).

77. Zhou, X. et al. Multiple functions of Osterix are required for bone growth and homeostasis in postnatal mice. Proc. Natl Acad. Sci. USA 107, 12919-12924 (2010).

78. Cheng, S., Xing, W., Zhou, X. \& Mohan, S. Haploinsufficiency of osterix in chondrocytes impairs skeletal growth in mice. Physiol. Genomics 45, 917-923 (2013).

79. Rodda, S. J. \& McMahon, A. P. Distinct roles for Hedgehog and canonical Wnt signaling in specification, differentiation and maintenance of osteoblast progenitors. Development 133, 3231-3244 (2006).
80. Chang, W., Tu, C., Chen, T. H., Bikle, D. \& Shoback, D. The extracellular calciumsensing receptor (CaSR) is a critical modulator of skeletal development. Sci. Signal. 1, ra1 (2008).

81. Rodriguez, L., Cheng, Z., Chen, T. H., Tu, C. \& Chang, W. Extracellular calcium and parathyroid hormone-related peptide signaling modulate the pace of growth plate chondrocyte differentiation. Endocrinology 146, 4597-4608 (2005).

82. Santa Maria, C. et al. Interplay between CaSR and PTH1R signaling in skeletal development and osteoanabolism. Semin. Cell Dev. Biol. 49, 11-23 (2016).

83. Tsonis, P. A. et al. A novel role of the hedgehog pathway in lens regeneration. Dev. Biol. 267, 450-461 (2004).

84. Hirai, T., Chagin, A. S., Kobayashi, T., Mackem, S. \& Kronenberg, H. M. Parathyroid hormone/parathyroid hormone-related protein receptor signaling is required for maintenance of the growth plate in postnatal life. Proc. Natl Acad. Sci. USA 108 191-196 (2011).

85. Hammond, C. L. \& Schulte-Merker, S. Two populations of endochondral osteoblasts with differential sensitivity to Hedgehog signalling. Development 136, 3991-4000 (2009).

86. Ingham, P. W. \& McMahon, A. P. Hedgehog signaling in animal development: paradigms and principles. Genes Dev. 15, 3059-3087 (2001).

87. Kim, E. J. et al. Ihh and Runx2/Runx3 signaling interact to coordinate early chondrogenesis: a mouse model. PLOS ONE 8, e55296 (2013).

88. Kobayashi, T. et al. Indian hedgehog stimulates periarticular chondrocyte differentiation to regulate growth plate length independently of PTHrP. J. Clin. Invest. 115, 1734-1742 (2005)

89. Long, F., Zhang, X. M., Karp, S., Yang, Y. \& McMahon, A. P. Genetic manipulation of hedgehog signaling in the endochondral skeleton reveals a direct role in the regulation of chondrocyte proliferation. Development 128, 5099-5108 (2001).

90. Maeda, Y. et al. Indian Hedgehog produced by postnatal chondrocytes is essential for maintaining a growth plate and trabecular bone. Proc. Natl Acad. Sci. USA 104, 6382-6387 (2007)

91. Shimoyama, A. et al. Ihh/Gli2 signaling promotes osteoblast differentiation by regulating Runx2 expression and function. Mol. Biol. Cell 18, 2411-2418 (2007).

92. Tu, X., Joeng, K. S. \& Long, F. Indian hedgehog requires additional effectors besides Runx2 to induce osteoblast differentiation. Dev. Biol. 362, 76-82 (2012).

93. Paul, S. et al. Ihha induces hybrid cartilage-bone cells during zebrafish jawbone regeneration. Development 143, 2066-2076 (2016).

94. Xing, W. et al. Thyroid hormone receptor-beta1 signaling is critically involved in regulating secondary ossification via promoting transcription of the thh gene in the epiphysis. Am. J. Physiol. Endocrinol. Metab. 310, E846-E854 (2016).

95. Takahashi, T. Overexpression of Runx2 and MKP-1 stimulates transdifferentiation of 3T3-L1 preadipocytes into bone-forming osteoblasts in vitro. Calcif. Tissue Int. 88, 336-347 (2011).

96. Gersbach, C. A., Byers, B. A., Pavlath, G. K. \& Garcia, A. J. Runx2/Cbfa1 stimulates transdifferentiation of primary skeletal myoblasts into a mineralizing osteoblastic phenotype. Exp. Cell Res. 300, 406-417 (2004).

97. Miyazaki, T. et al. Inhibition of the terminal differentiation of odontoblasts and their transdifferentiation into osteoblasts in Runx2 transgenic mice. Arch. Histol. Cytol. 71, 131-146 (2008).

98. Pacifici, M. et al. Hypertrophic chondrocytes. The terminal stage of differentiation in the chondrogenic cell lineage? Ann. N. Y. Acad. Sci. 599, 45-57 (1990).

99. van der Kraan, P. M. \& van den Berg, W. B. Chondrocyte hypertrophy and osteoarthritis: role in initiation and progression of cartilage degeneration? Osteoarthritis Cartilage 20, 223-232 (2012).

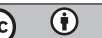

Open Access This article is licensed under a Creative Commons Attribution 4.0 International License, which permits use, sharing, adaptation, distribution and reproduction in any medium or format, as long as you give appropriate credit to the original author(s) and the source, provide a link to the Creative Commons license, and indicate if changes were made. The images or other third party material in this article are included in the article's Creative Commons license, unless indicated otherwise in a credit line to the material. If material is not included in the article's Creative Commons license and your intended use is not permitted by statutory regulation or exceeds the permitted use, you will need to obtain permission directly from the copyright holder. To view a copy of this license, visit http://creativecommons. org/licenses/by/4.0/.

(c) The Author(s) 2018 